\title{
STUDIES ON PRODUCTION OF BIODIESEL FROM WASTE COOKING OIL USING SOLID WASTE AS CATALYST
}

\section{RAJAM BHUKYA}

Department of Chemical Engineering, University College of Technology, Osmania University, Hyderabad, Telangana, India

ABSTRACT
Production of Biodiesel from waste cooking oil is considered one of the interesting processes in recent years. In this
paper, Catalyst was prepared from solid waste materials, fly ash, and used for the production of biodiesel from waste
cooking oil. Physico-chemical characterization of the prepared catalyst was done using FTIR, XRD and SEM.
Performance evaluation of prepared catalyst was done for the maximum yield of biodiesel by varying temperature,
reactants molar ratio (methanol to WCO) and loading of catalyst were studied and analyzed. The maximum conversion
of $47 \%$ WCO was obtained at $60^{\circ} \mathrm{C}$ in 90 min for molar ration of 1:9 (WCO to methanol) at loading of catalyst is $1.5 \%$
by weight of reaction mixture. The reaction was carried out till the equilibriumobtained.
KEYWORDS: Waste Cooking Oil, Flyash Catalyst, Biodiesel, FTIR, XRD, SEM, Transesterification Reaction

Received: Jun 09, 2020; Accepted: Jun 29, 2020; Published: Jul 31, 2020; Paper Id.: IJMPERDJUN2020549

\subsection{INTRODUCTION}

There are many issues associated with the application of the petroleum based fuels. Higher depletion rates of resources, increased cost for production, increased pollution problems are some of them. All these features are motivating the technologists to search for the alternative renewable fuels [1]. On the other hand, huge quantities of animal fats and waste cooking oils (WCO) are abundantly available and managing of such fats and oils is a very significant challenge due to their disposal problems. The used vegetable oil, it is other way called as waste cooking oil, is one of the best cost-effective options to harvest biodiesel. Subsequently, one of the major concerns for the manufacture of biodiesel is the cost of feedstock; use of WCO considerably increases the economic feasibility of biodiesel manufacture [2]. Transesterification with a catalyst is the reaction of oil with an alcohol $(\mathrm{ROH}, \mathrm{R}=\mathrm{CH} 3$ or C2H5 and etc.) and a catalyst to produce esters and glycerol [3]. Methanol is basically employed due to low cost, physical and chemical advantages such as polar and smallest chain alcohol. Transesterification can happen depending on the oil at different temperatures. On the other hand, a higher temperature apparently affects the reaction rate and ester's yield. The product of the transesterification reaction contains primarily of esters, glycerol, and traces of alcohol, catalyst, triglycerides, Diglycerides, and Monoglycerides [4]. Among the methods, transesterification is the best adoptable process, which is an organic process in which an ester is transformed into the alkoxy molecule. The chemical reaction is shown in Scheme (I). 


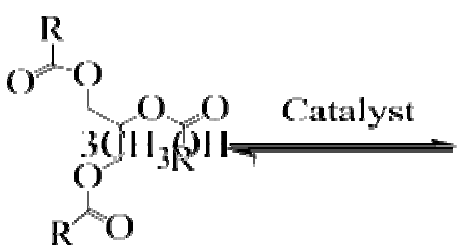

'Iriglyceride 3 Methanol<smiles>OCC(O)CO</smiles>

(ilycerol

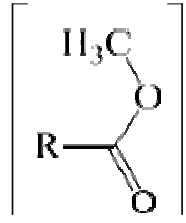

3 Methyl Fisters

Also, the preparation of catalysts of heterogeneous from waste materials has been becoming progressively more popular from the last two decades. Some of the most interesting resources are waste biomass from agriculture such as inedible oils, animal waste and Flyash [5]. Application of waste materials is highly advantageous in view of financial or conservation costs accompanying with their discarding. The problems involved with homogeneous catalysts such as removal of costly catalysts, separation of products, longer operational time will be rightly answered by heterogeneous catalysts. Unlike to homogeneous catalysts, solid heterogeneous catalysts have the advantages of ease of separation from the reaction mixture after the reaction completed and ease of regeneration of the material forreuse[6].The main objective of present paper is to develop the heterogeneous catalysts from solid waste materials such as flyash and to study the performance of the heterogeneous catalyst towards the biodiesel synthesis from WCO.

\subsection{MATERIALS AND METHODOLOGY}

The catalyst is prepared from solid waste material such as fly ash obtained from Thermal Power Plant (NTPC). The materials were collected from where they were available at low cost or zero cost. The solvents and reagents used for the preparation of solid catalysts and for reaction studies were A. R. grade (99.9\%). The solvents and reagents were procured from M/S Sd Fine- Chem Limited, Mumbai, India.

\subsection{Method of Catalyst Preparation}

Fly ash is a product of coal and contains fine particles. In modern coal based power plants, fly ash is usually prevented by electrostatic precipitators (ESP) or other particle filtration equipment like bag filters after the flue gases reach the chimneys. The composition of fly ash could differ significantly depending upon the source of the coal where it burned. Mostly, the fly ash includes considerable amounts of silicon dioxide ( $\mathrm{SiO}$ ) (both amorphous and crystalline), aluminum oxide (Al2O3), calcium oxide $(\mathrm{CaO})$ and etc. Various methods were developed to prepare catalysts using fly ash. Wet impregnation method was adopted to prepare the solid catalyst from solid waste materials. In this, for $10 \mathrm{gm}$ of fly ash dry sample, $10.2 \mathrm{~mL}$ of $1 \mathrm{~N}$ aqueous solution of potassium nitrate is added slowly with constant manual stirring until the homogeneous mixture is attained. Then, the excess water is driven off using the rotary evaporator. After drying at $100 \mathrm{oC}$ till complete removal of water, the solid mixture is calcined at a temperature of $500{ }^{\circ} \mathrm{C}$ for $5 \mathrm{~h}$ in an ambient air flow. The obtained dry fly ash-KNO3 catalyst powder was characterized by XRD, SEM and FTIR technics. The prepared fly ashKNO3 catalyst powder was directly used for the production of biodiesel from WCO. The high temperature furnace was used to prepare fly ash-KNO3 catalyst. The high temperature furnace was shown in Figure (2). The fly ash powder used as received condition for synthesis the catalyst and after heat treatment condition (fly ash-KNO3 catalyst) was shown in Figure(3). 


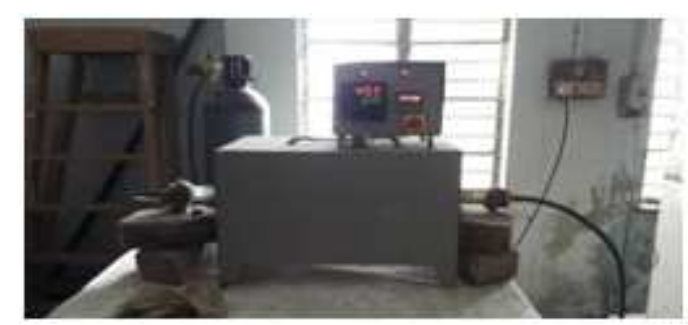

Figure 2: High temperature furnace used to prepare fly ash catalysts.
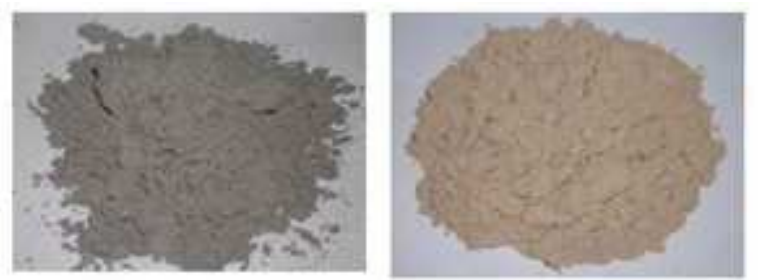

Figure 3: The fly ash used for synthesis (a) as it is received condition and (b) After heat

\subsection{Characterization of Catalysts}

The primary objective of catalyst characterization is to study the relationship among physical and chemical properties as well as its activity. It is important for designing and a process optimization. The characterization is required and necessary to observe the variations in chemical and physical properties of a catalyst in the course of preparation, activation and reaction steps for well understanding and control of quality. The prepared catalyst is characterized by XRD, SEM and FTIR.The prepared samples were analyzed by a Siemens $500 \mathrm{X}$ - ray powder diffractometer in a flat plane geometry with a source of $\mathrm{Cu}-\mathrm{K} \alpha$ of wavelength, $1.5406 \AA$. The XRD spectra were recorded in the region of 10-80 o(2 $\theta)$ in steps of 0.001 o with a counting time of $1 \mathrm{~s}$ in each step. The specifications of the instruments for the XRD analysis are given in Table 1.

Table 1: Measurement Conditions

\begin{tabular}{|l|l|}
\hline \multicolumn{1}{|c|}{ X-Ray tube of target } & \multicolumn{1}{c|}{$\mathbf{C u}-\mathbf{K} \boldsymbol{\alpha}$} \\
\hline Voltage & $40 \mathrm{KV}$ \\
\hline Current & $30.0 \mathrm{~mA}$ \\
\hline \multicolumn{2}{|c|}{ slits } \\
\hline Divergence slit & $1.0^{\mathrm{O}}$ \\
\hline Scatter slit & $10.0^{\mathrm{O}}$ \\
\hline Receiving slit & $0.3 \mathrm{~mm}$ \\
\hline Scanning & $\theta-2 \theta$ \\
\hline Scan range & $10.0-80.0^{\mathrm{O}}$ \\
\hline Scan mode & continuous scan \\
\hline Scan speed & 2.0 degree/min \\
\hline sample pitch & 0.02 degree \\
\hline Present time & $0.6 \mathrm{sec}$ \\
\hline
\end{tabular}

The powder XRD patterns of fly ash-KNO3 catalyst were presented in the Figure 4. The compound exhibited the characteristic peaks of crystalline phases such as silica $(\mathrm{SiO} 2)$, calcium carbonate $(\mathrm{CaCO} 3)$, alumina $(\mathrm{Al} 2 \mathrm{O} 3)$ and iron oxide ( $\mathrm{Fe} 2 \mathrm{O} 3$ ). The predominant phase was quartz ( $\mathrm{SiO} 2)$ with main peak at $23.16^{\circ}(2 \theta)$ and other peaks of $\mathrm{CaCO}$, $\mathrm{Al} 2 \mathrm{O} 3$ and $\mathrm{Fe} 2 \mathrm{O} 3$ were identified as shown in the Figure 4. From the XRD result of fly ash-KNO3 catalysts, it has the crystalline phases of quartz, magnetite and mullite. The hematite peak was not found due to the high concentration of magnetite. 


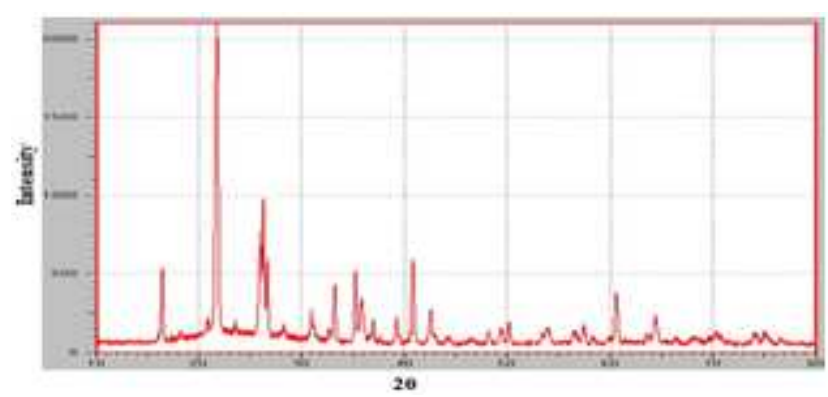

Figure 4: XRD graph of Fly ash-KNO3 Catalyst.

\subsection{SEM Analysis}

SEM is useful in obtaining morphology of the unknown components. The morphology of the fly ash-KNO3, egg shell and sodium bentonite catalysts synthesized in the present work was examined by using a Hitachi X-650 scanning electron microscope (SEM) analyzer equipped with a CDU lead detector at $25 \mathrm{kV}$. The prepared samples were placed on aluminum pegs and coated with a gold thin film to allow them conductive. The SEM scans with a focused electron beam onto a surface to produce an image. The electrons from the beam interact with the prepared sample, generating a number of signals that can be used to attain images about the surface morphology. The SEM can produce three-dimensional images. The resolution is $0.2 \mathrm{~nm}$ which is 1000 times more than a light microscope. A typical SEM photograph of fly ash-KNO3 catalyst is shown in the Figures 5 and 6. It shows the irregular morphology of fly ash-KNO3. It provides three dimensional data on an atomic or near atomic scale for varying resolutions of 500, 100, 50 and $10 \mu \mathrm{m}$.

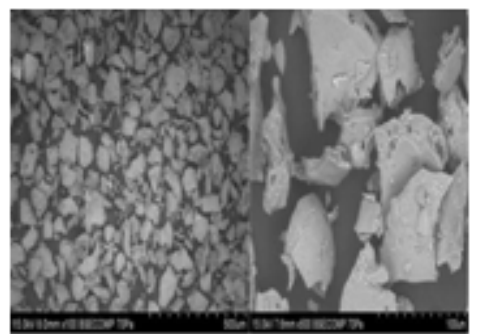

Figure 5: SEM photographs of fly ash-KNO3 catalyst with 500 and $100 \mu \mathrm{m}$ resolutions.

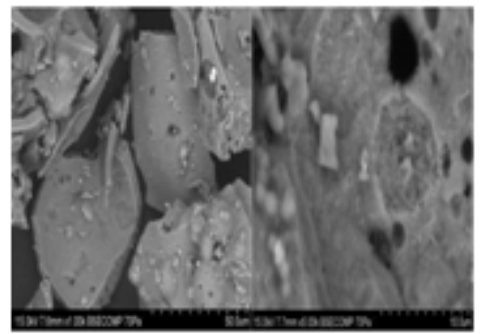

Figure 6: SEM photographs of fly ash-KNO3 catalyst with 50 and $10 \mu \mathrm{m}$ resolutions.

\subsection{Fourier Transform Infrared Spectroscopy (FT-IR)}

The FT-IR was used to study the changes in a material and also give data about the functional of a sample. It requires practically sample preparation with potassium bromide as a pellet. However, around $15 \mathrm{mg}$ of the prepared catalysts sample is employed in the attenuated total reflectance (ATR) sample holder of a Perkin Elmer Spectrum 100 FT-IR spectrometer. The samples were recorded in the range of 4000-400 cm-1, baseline was corrected and the spectra were smoothened. The FTIR bands from 900 to $1200 \mathrm{~cm}-1$ were recognized as various types of $\mathrm{Si}-\mathrm{O}$ and $\mathrm{Si}-\mathrm{O}-\mathrm{Si}$ stretching vibrations. The other bands between 450 and $900 \mathrm{~cm}-1$ in the spectra of fly ash-KNO3 were gave the typical Si-O and Si- 
O-Al bending modes. The FTIR patterns of fly ash-KNO3 catalyst is shown in Figure7.

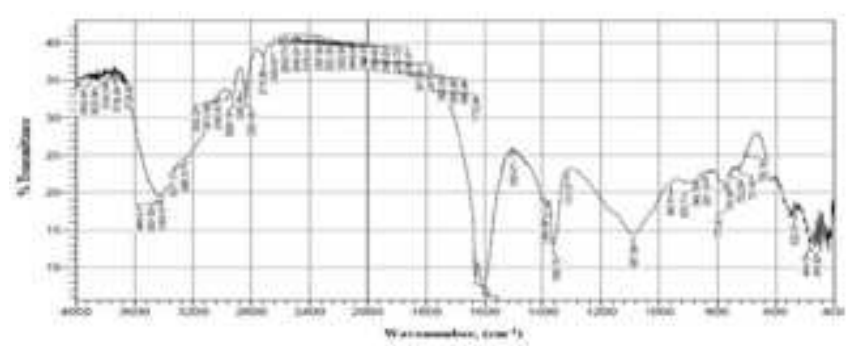

Figure 7: FT-IR patterns of fly ash-KNO3 catalyst.

\subsection{EXPERIMENTAL}

The prepared heterogeneous catalyst is used for the production of biodiesel from WCO. It has a series of three sequential reversible reactions where triglycerides (TGs) are converted to diglycerides (DGs) and DGs are converted to monoglycerides (MGs) and MGs are converted to glycerol. In each step an ester is formed and thus three ester molecules are generated from one molecule of TG. At the initial stage of the reaction, there is a need of vigorous mixing of oil. So, the oil and the methanol get uniformly mixed. However, as the process results the formation of the product, slight mixing is required to facilitate the settling down of glycerol, so that reactions can continue in the upper layer of methanol and oil. The stoichiometry of transesterification of triglyceride (WCO) with methanol was shown in Scheme (I). The stoichiometric relation between the oil and the increase there suitable to The transesterification reaction is accelerated by a catalyst. Various types of catalysts including homogeneous (such as $\mathrm{HCl}, \mathrm{H} 2 \mathrm{SO} 4$ ) [7] or heterogeneous catalysts (metal based catalysts like Zeolite supported alumina) are employed for the process. The advantages of heterogeneous catalysts like ease of separation of products attracting the researchers to go with heterogeneous catalysis. As the reaction is reversible in nature excess alcohol is used to shift the reaction towards the products. Flyash catalytic performance is studied in synthesis of biodiesel from WCO. All the experiments were performed at atmospheric pressure and the effect of several variables were studied on the conversion of WCO to biodiesel. The different parameters and their range used in the experiments were given in Table 2.

Table 2: List of Different Parameters and Their Range in the Experiments

\begin{tabular}{|l|c|}
\hline \multicolumn{1}{|c|}{ Parameters } & Range \\
\hline Temperature & 40,50 and $60{ }^{\circ} \mathrm{C}$ \\
\hline Mole Ratio (Alcohol to WCO) & $6: 1,9: 1$ and $12: 1$ \\
\hline Catalyst Loading (\% of reaction mixture weight) & $0.5,1.0$ and 1.5 \\
\hline Rotational Speed & $800 \mathrm{rpm}$ \\
\hline
\end{tabular}

\subsection{Experimental Setup}

A magnetic stirrer assembled with heating mantle (make REMI) was used to carry out the reaction. A one liter three necked round bottom flask was used as reactor which was equipped with a reflux condenser of water-cooling at the center neck. One neck of the flask was used as thermo well for a thermometer and the other neck is closed with a dummy cap through which reactants to be fed and mid reaction samples were collected for analysis. The entire apparatus was placed in an oil bath to maintain the reaction temperatures with $\pm 2{ }^{\circ} \mathrm{C}$ accuracy. The experimental system was shown in Figure 8 .

In a usual run, certain amount of WCO and calculated amounts of catalyst taken into the RB flask. The whole contents were heated up to required temperature gradually. Room temperature water supply was arranged across the reflux 
condenser to arrest the escaping of vapors which were formed during the reaction. Then, after attaining the pre specified temperature, specified amount of methanol was introduced to the flask and the instant was read as zero time. The entire process was carried out at ambient pressure. For every $15 \mathrm{~min}$ of time interval, the stirrer was switched off and the mixture were permitted to settle for 2-3 min. After settling the reaction mixture, around $5 \mathrm{~mL}$ of reaction mixture from top layer was collected carefully using a pipette so that neither bottom layer nor catalyst particles are present in the sample. The sample was transferred to an ice cold chamber and for that sample $5 \mathrm{~mL}$ of isopropyl alcohol was added and analysis was carried out immediately. The process was continued for $90 \mathrm{~min}$ and every $15 \mathrm{~min}$ sample was collected and analyzed immediately. The composition of WCO was given in Table 3.

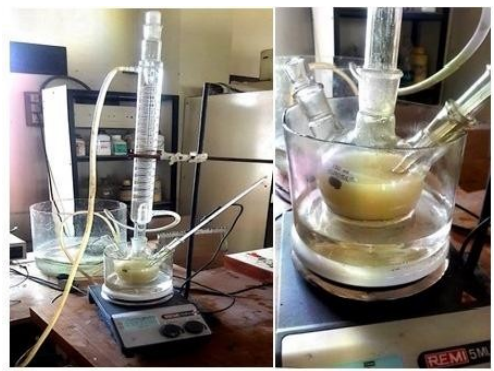

Figure 8: Experimental setup for biodiesel synthesis from WCO

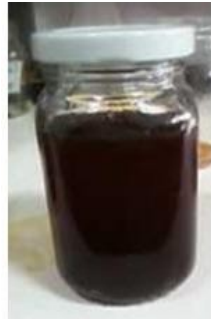

(a)

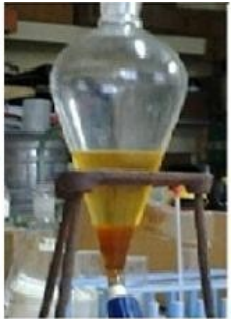

(b)
Figure 9: (a) Waste cooking oil, (b) Formation of two layers of the reaction mixture in the biodiesel production process

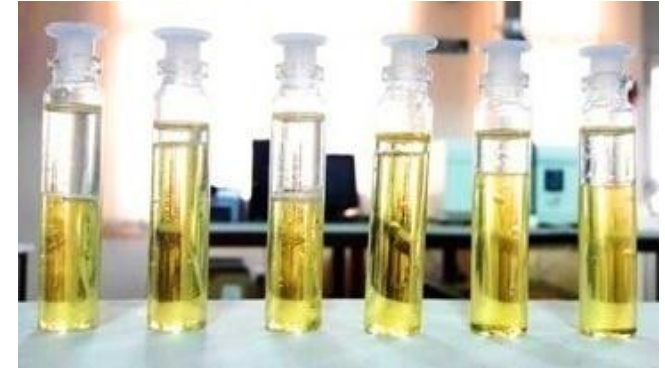

Figure 10: Samples of the reaction mixture collected at different time intervals. (15- 90 min)

Figure 8: Experimental setup for biodiesel synthesis from WCO Figure 9: (a) Waste cooking oil, (b) Formation of two layers of the reaction mixture in the biodiesel production process $\quad$ Figure 10 : Samples of the reaction mixture collected at different time intervals. (15-90 $\mathrm{min})$

Table 3: Composition of waste cooking oil

\begin{tabular}{|c|c|c|}
\hline $\begin{array}{c}\text { Name of the fatty } \\
\text { acid }\end{array}$ & $\begin{array}{c}\text { Molecular } \\
\text { formula }\end{array}$ & $\begin{array}{c}(\%) \\
\text { Composition }\end{array}$ \\
\hline Stearic & $\mathrm{C}_{18 \mathrm{H} 36 \mathrm{O} 2}$ & 04.5 \\
\hline Oelic & $\mathrm{C} 18 \mathrm{H} 34 \mathrm{O} 2$ & 21.1 \\
\hline Linoleic & $\mathrm{C} 18 \mathrm{H} 32 \mathrm{O} 2$ & 66.2 \\
\hline Eicosanioc & $\mathrm{C} 20 \mathrm{H} 40 \mathrm{O} 2$ & 00.3 \\
\hline Others & & 07.9 \\
\hline
\end{tabular}

After completion of the reaction, the reaction mixture was shifted to a separating funnel and permitted to separate in to the two layers. The top layer (biodiesel layer) was recovered and analyzed. The remaining methanol was removed by using rotary evaporation system. Data was collected to determine the conversion of WCO. The experiments were performed to study the effect of various parameters for example temperature, molar ratio and loading of a catalyst. Formation of two layers of the reaction mixture in the biodiesel production process was shown in Figure 9. The reaction mixture samples were collected at different time intervals was shown in Figure 10.

\subsection{RESULTS AND DISCUSSIONS}

Number of experiments was conducted for the reaction system of biodiesel production from WCO. The effect of the various parameters such as temperature, molar ratio of the reactants and catalyst loading were studied and were presented 
in the following sections. The effect of the various parameters over the progress of reaction/conversion were studied, data collected and the results were analyzed.

\subsection{Effect of Temperature}

As the reaction rate constant is a function of a temperature, it is significant to study the effect of temperature on the formation of biodiesel from the WCO. In order to find the best temperature, the reaction was carried out at various molar ratios of methanol to oil at different temperatures, using three different catalysts. The temperature effect was studied at catalyst loading of $1.0 \%$ and molar ratio of WCO to methanol is 1:9. As shown in Figure 13, the reaction rate was slow at lower temperatures and with increase in temperature from 40 to $60{ }^{\circ} \mathrm{C}$, a resultant increase in the conversion of the WCO was observed. But at the temperatures of $70{ }^{\circ} \mathrm{C}$ and above, there is fractional decrease in the conversion of WCO. It may be due to the fact that the boiling point of methanol is around $65{ }^{\circ} \mathrm{C}$ and reaction above this temperature might cause the methanol to vaporize into gas phase leading to low conversion of WCO. In addition, higher temperatures would increase the risk of saponification. Thus, the highest reaction temperature was limited to $60{ }^{\circ} \mathrm{C}$ for the transesterification of WCO and methanol. The effect of temperature at molar ratio of WCO to methanol is 1:9, catalyst loading is $1.0 \%$ based on weight percent of the solution mixture was shown in Figures 10 and12.

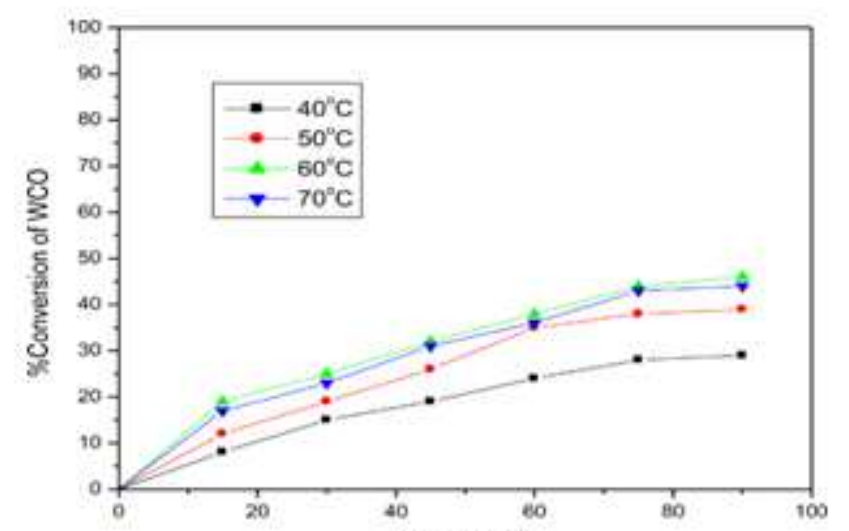

Figure 11: Effect of temperature for the conversion of $\mathrm{WCO}$ with the catalyst fly ash-KNO3 for mole ration= 9 catalyst loading=1\%

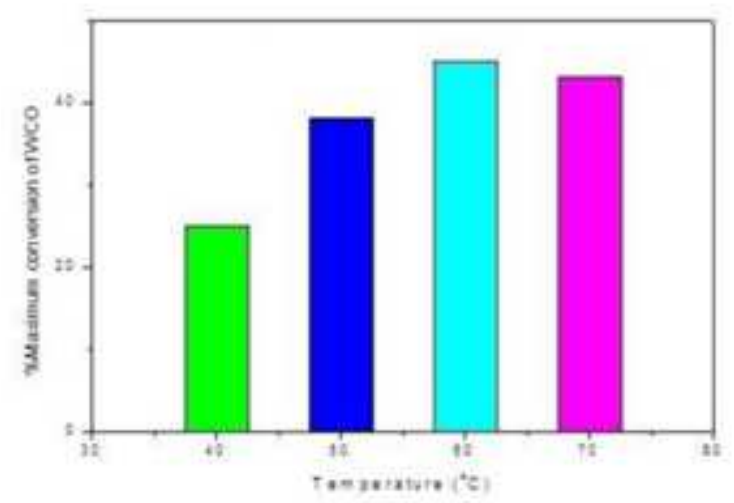

Figure 12: Comparative plot of maximum conversions of WCO with the temperature For the1.5\% fly ash-KNO3 catalyst.

\subsection{Effect of Molar Ratio of WCO to Methanol}


One of the most significant factors that alter the yield of product is the molar ratio of methanol to WCO. This parameter is imperative for showing the catalyst performance. While the stoichiometric molar ratio of methanol to triglycerides for transesterification is $3: 1$, excess molar ratios are employed to increase the solubility and to improve the contact among the triglyceride and alcohol particles. Precisely, it has furthermore been evidenced that the use of excess alcohol is a good selection in increasing the reaction rate of the transesterification where heterogeneous catalysts are regarded.

The effect of the molar ratio of methanol to WCO, on the conversion of WCO was studied with the three catalysts and varying loading of a catalyst. Figures 13 to 15 represent the change in the percentage conversions of WCO at 0.5 , 1.0 and $1.5 \%$ catalyst loadings of fly ash-KNO3. From Figures 13 to 15, it was observed that with increasing in the mole ratios of reactants, the conversion of $\mathrm{WCO}$ was also increasing. At higher loadings of the catalyst, the effect of mole ratio is more than that of lower loadings.

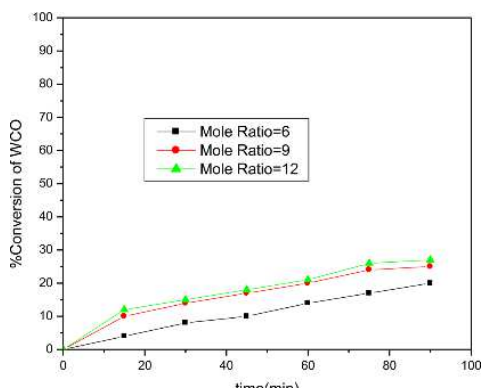

Figure 13: Effect of molar ratio for the conversion of WCO for $0.5 \%$ loading offly ash-KNO3 at $60^{\circ} \mathrm{C}$.

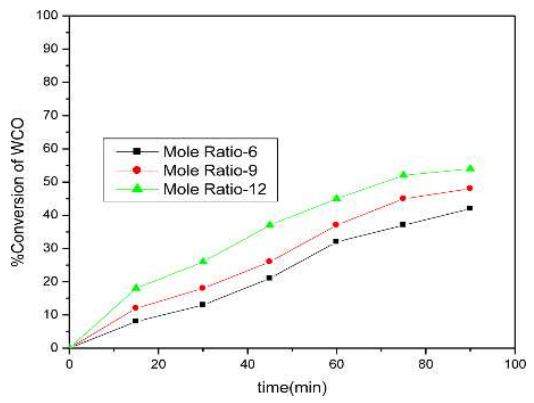

Figure 14: Effect of molar ratio for the conversion of WCO for $1.5 \%$ catalyst loading of fly ash-KNO3 at 60

${ }^{\circ}$ C.

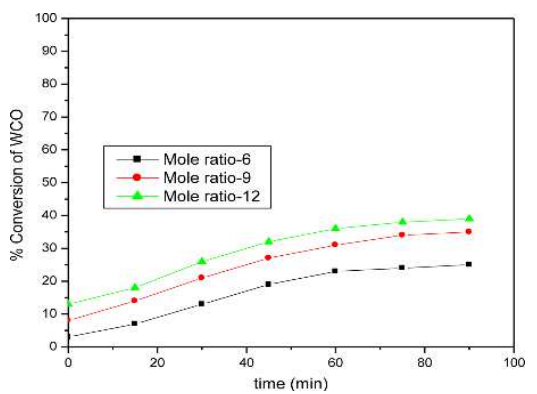

Figure 15: Effect of molar ratio for the conversion of WCO for $1.0 \%$ loading of fly ash-KNO3 at $60{ }^{\circ} \mathrm{C}$.

\subsection{Effect of Catalyst Loading}

For the reaction, larger amounts of catalyst lead to higher yield of esters in a lesser reaction time. In the reaction, three amounts of catalyst loadings are tested i. e, 0.5, 1.0 and $1.5 \%$ of total reaction mixture weight for the three catalysts. The observations are presented in the Figure16. From the figure, it was observed that with increase in catalyst loadings, the 
conversions are increased for the three catalysts. For the $1.5 \%$ catalyst loading of the three catalysts was resulted in maximum conversions of WCO. From the above study, it could be concluded that the fly ash - KNO3 catalysts gave just $47 \%$ conversion of the WCO respectively.

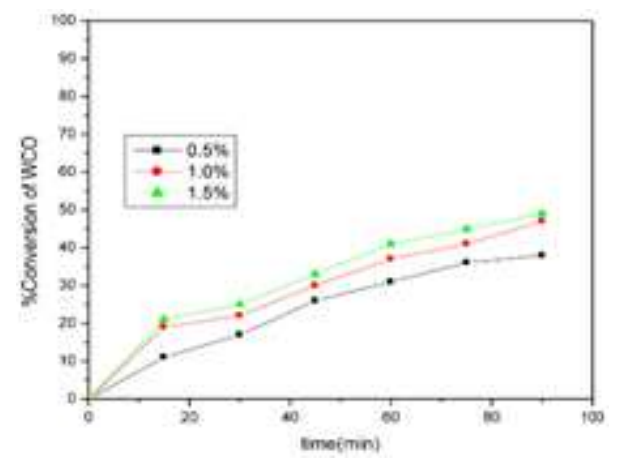

Figure 16: Effect of catalyst loading for the conversion of WCO with fly ash-KNO3 catalyst at $60{ }^{\circ} \mathrm{C}$ and molar ratio of methanol to WCO is 9:1.

\subsection{CONCLUSIONS}

The production of the biodiesel from WCO with methanol was carried out with Flyash-KNO3 catalyst in conventional batch reactor. The prepared Catalyst was characterized by FTIR, XRD and SEM. Performance of the prepared catalysts was evaluated by varying the temperature, molar ratios and catalyst loading for the production of biodiesel from WCO. The conversion of WCO increased with increasing the temperatures, molar ratio and catalyst loading. The maximum conversion of $47 \%$ WCO was obtained at temperature of $60^{\circ} \mathrm{C}$, molar ratio of 9:1 (methanol to WCO) within 90 min at 1.5 $\%$ catalyst loading.

\section{REFERENCES}

1. Heather L. MacLeana, Lester B. Lave, "Evaluating automobile fuel/propulsion system technologies", Progress in Energy and Combustion Science, 29 (2003)1-69.

2. SrinathSuranani, YadagiriMaralla, Shekhar M. Gaikwad, Shirish H. Sonawane, "Process intensification using corning ${ }^{\circledR}$ advanced-flow ${ }^{\mathrm{TM}}$ reactor for continuous flow synthesis of biodiesel from fresh oil and used cooking oil”, Chemical Engineering \& Processing: Process Intensification 126 (2018)62-73.

3. Sharma, Shiv Kumar, et al. "Performance evaluation of diesel engine using biodiesel fuel derived from waste cooking refined soyabean oil." International Journal of Mechanical and Production Engineering Research and Development (IJMPERD) 7.5 (2017): 103-110.

4. Ying Xia Li and Bing Xue Dong, “Optimization of Lipase-Catalyzed Transesterification of Cotton Seed Oil for Biodiesel Production Using Response Surface Methodology”, Brazilian Archives Of Biology and Technology, 59, (2016)1-7.

5. Deepak Verma, Janmit Raj, Amit Pak, Manish Jain, "A critical review on production of biodieselfromvariousfeedstocks",JournalofScientificandInnovativeResearch,5,2,(2016)51-58.

6. Carlos A. Guerrero F., Andres Guerrero-Romero, Fabio E. Sierra, “Biodiesel Production from Waste Cooking Oil”, Biodiesel -Feed stocks and Processing Technologies, Edited by Dr. Margarita Stoytcheva, (2011) 23 -45.

7. Bhaskar, K., et al. "Effect of dimethoxy-methane (C3H8O2) additive on emission characteristics of a diesel engine fueled with biodiesel." International Journal of Mechanical and Production Engineering Research and Development 8.1 (2018): 399-406. 
8. George Anastopoulos, YpatiaZannikou, StamoulisStournas, Stamatis Kalligeros, "Transesterification of Vegetable Oils with Ethanol and Characterization of the Key Fuel Properties of Ethyl Esters”, Energies, 2 (2009) 362 -376.

9. Girish, C. R. "Catalyst preparation from various naturally available waste Materials for biodiesel production: a review." International Journal of Mechanical and Production Engineering Research and Development 9.6 (2019): 117-130.

10. James A. Bennett, Karen Wilson, Adam F. Lee, "Catalytic applications of waste derived materials, Journal of Materials Chemistry A, 4, 6 (2016) 3617 -3637.

11. M. C. Math, SudheerPrem Kumar, Soma V. Chetty, "Technologies for biodiesel production from used cooking oil - A review”, Energy for Sustainable Development, 14, 4 (2010)339-345.

12. Hymavathi, D., G. Prabhakar, and B. B. Sarath. "Biodiesel production from vegetable oils: an optimization process." Int J Chem Petrochem Technol, 4 (2), 2130 (2014).

13. B. Rajam, Ch. Venumadhav, P. S. Saiprasad, Ch. Sailu and V. V. Basavarao, "Thermodynamic parameter Evaluation and Reaction Studies for butanol esterification process in presence of sodium- bentonite catalyst", Journal of Engineering and Technology, 3 (1) (2015)1-10. 